\title{
THE PRACTICAL APPLICATION OF UAV-BASED PHOTOGRAMMETRY UNDER ECONOMIC ASPECTS
}

\author{
M. Sauerbier ${ }^{\text {a, } * \text { E. Siegrist }}{ }^{b}$, H. Eisenbeiss ${ }^{c}$, N. Demir ${ }^{c}$ \\ ${ }^{a}$ MFB GeoConsulting GmbH, Eichholzstrasse 23, Messen, Switzerland - sauerbier@ mfb-geo.com \\ b omnisight GmbH, Balz-Zimmermann-Strasse 34, Zurich, Switzerland - emil.siegrist@omnisight.ch \\ ${ }^{c}$ Institute of Geodesy and Photogrammetry, ETH Zurich, Wolfgang-Pauli-Strasse 15, Zurich, Switzerland - \{ehenri, \\ nusret.demir\}@geod.baug.ethz.ch
}

Commission I, WG I/V

KEY WORDS: Adjustment, Matching, Photogrammetry, Point Cloud, Surface, Test, UAVs

\begin{abstract}
:
Nowadays, small size UAVs (Unmanned Aerial Vehicles) have reached a level of practical reliability and functionality that enables this technology to enter the geomatics market as an additional platform for spatial data acquisition. Though one could imagine a wide variety of interesting sensors to be mounted on such a device, here we will focus on photogrammetric applications using digital cameras. In praxis, UAV-based photogrammetry will only be accepted if it a) provides the required accuracy and an additional value and b) if it is competitive in terms of economic application compared to other measurement technologies. While a) was already proven by the scientific community and results were published comprehensively during the last decade, b) still has to be verified under real conditions.

For this purpose, a test data set representing a realistic scenario provided by ETH Zurich was used to investigate cost effectiveness and to identify weak points in the processing chain that require further development. Our investigations are limited to UAVs carrying digital consumer cameras, for larger UAVs equipped with medium format cameras the situation has to be considered as significantly different.

Image data was acquired during flights using a microdrones MD4-1000 quadrocopter equipped with an Olympus PE-1 digital compact camera. From these images, a subset of 5 images was selected for processing in order to register the effort of time required for the whole production chain of photogrammetric products.

We see the potential of mini UAV-based photogrammetry mainly in smaller areas, up to a size of ca. 100 hectares. Larger areas can be efficiently covered by small airplanes with few images, reducing processing effort drastically. In case of smaller areas of a few hectares only, it depends more on the products required. UAVs can be an enhancement or alternative to GNSS measurements, terrestrial laser scanning and ground based photogrammetry. We selected the above mentioned test data from a project featuring an area of interest within the practical range for mini UAVs. While flight planning and flight operation are already quite efficient processes, the bottlenecks identified are mainly related to image processing. Although we used specific software for image processing, the identified gaps in the processing chain today are valid for most commercial photogrammetric software systems on the market. An outlook proposing improvements for a practicable workflow applicable in projects in private economy will be given.
\end{abstract}

\section{INTRODUCTION}

During the last decade, research on UAVs has increased strongly in terms of systems, sensor integration and data processing as well as in different scientific disciplines such as robotics, computer vision, geomatics and others more. Here, we focus on photogrammetric application of UAVs, a sector that has achieved the transition from research towards practical deployment of the UAVs in private economy and in the public sector from a technical point of view.

Nevertheless, UAV photogrammetry faces competing technologies already established in the market, such as GNSS measurement, terrestrial laser scanning and photogrammetry, and airborne photogrammetry and laser scanning using airplanes or helicopters and medium or large format cameras. The goal of this paper is to investigate the economic potential of UAV photogrammetry in competition with the above mentioned technologies. In the following, only the application of UAVs equipped with digital optical sensors will be considered. Additionally, the added value introduced by UAVs and its significance for typical customers will be exemplified and based on a specific UAV system, sensor and photogrammetric software, the potential for improvement in order to achieve an optimised workflow will be proposed.

\subsection{UAV photogrammetry in practical application}

Recently, several private companies offer photogrammetric products produced by means of UAV-based imagery for certain applications, mainly these are DSMs and orthoimages. Furthermore, monitoring or change detection often plays a role, e.g. detection of new buildings, changes at forest areas, gravel pits or waste sites were so far identified as potential applications. Further and more specific applications can be found in the scientific literature (Eisenbeiss et al., 2005, Lambers et al., 2007, Reidelstuerz et al., 2007, Grenzdörffer et al., 2008, Eisenbeiss, 2009, Niethammer et al., 2009, Vogler et al., 2009).

Regarding system reliability, quality and accuracy of the derived products, technical practicability and handling of

\footnotetext{
* Corresponding author.
} 
modern UAVs, such systems are technically ready for deployment in photogrammetric projects in private economy. However, certain factors still hamper the processing chain from being optimal and therefore lead to challenges. First of all, no common standard for navigation data formats was accomplished so far such that each UAV generates its own format which in turn requires time consuming data conversion when importing these data into photogrammetric software.

\subsection{Customer requirements}

Based on customer requests from 2010 and 2011 concerning possible projects, we identified a general interest in UAV technology. These were mainly related to gravel pits and waste sites with the goal to measure changes in volume and for documentation of the site by means of additional imagery. It is expected that the Swiss administration will enact a law in 2012 that requests gravel pit carriers to additionally document changes by means of images every 3 months. This would support the application of UAVs for documentation and data acquisition tasks significantly due to the added value. Both, data for volumetric change calculation and documentation could be acquired during one campaign.

\subsection{Aim of the study}

The aim of the study was to evaluate the whole workflow from data acquisition to photogrammetric products using the Leica Photogrammetry Suite (LPS) software developed by Erdas in order to verify if UAV photogrammetry can be economically competitive. Furthermore, the time effort required for the workflow should be investigated in order to be able to predict it already reliably in the planning phase of new projects.

The focus was on the registration of time needed for all involved processing steps to identify possible bottlenecks and improvements. The system costs were not included into this study, although they vary a lot. Instead, we concentrate on service costs which mainly depend on operators' salaries, therefore the focus is on time efforts that have to be invested.

\section{PROCESSING OF A TEST DATA SET}

\subsection{Description of the test area}

The test site is a gravel pit carried by the Lötscher Kies \& Beton AG (Lötscher, 2011) in Ballwil near the city of Lucerne, $\mathrm{CH}$, where volumetric changes need to be calculated on a regular basis. The area of interest for our test comprised about 1.08 hectares $\left(120 \times 90 \mathrm{~m}^{2}\right)$. The height values differ between ca. $480 \mathrm{~m}$ to $506 \mathrm{~m}$ above mean sea level.

\subsection{Data Acquition}

Data acquisition was accomplished by a team of ETH Zurich (Institute of Geodesy and Photogrammetry) at 2 different periods, one in 2009 and one in 2010 in order to enable the detection of volumetric changes between both acquisition dates. While in 2009 only one camera was deployed, in 2010 photogrammetric flights with 3 different cameras mounted on a microdrones MD4-1000 quadrocopter (microdrones, 2011) were conducted (figure 1). The cameras were an Olympus EP-1, a Lumix FX 35 and a Sigma DP-1. For this study, only the 2010 images acquired using the Olympus EP-1 were used due to the fact that the best results were obtained with this camera in an earlier processing with a different purpose by ETH Zurich (figure 2). The acquired block consists of 26 images with $70 \%$ overlap in flight direction and $20 \%$ overlap between the stripes. From this block, a subset of 10 images was used for this study. For this part of the workflow, a time effort of $1 / 2$ day for the UAV operator was required and an additional $1 / 2$ day for 1 person conducting the GCP measurements. The GCPs were signalised with white circular targets.

For georeferencing, 11 ground control points (GCP) were determined in the Swiss coordinate system using differential GNSS measurements.

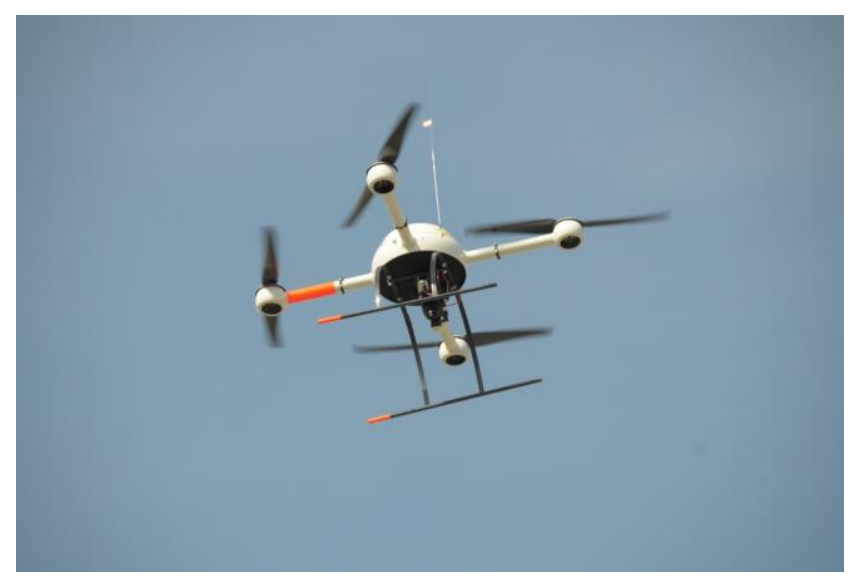

Fig. 1: The microdrones MD4-1000 quadrocopter.

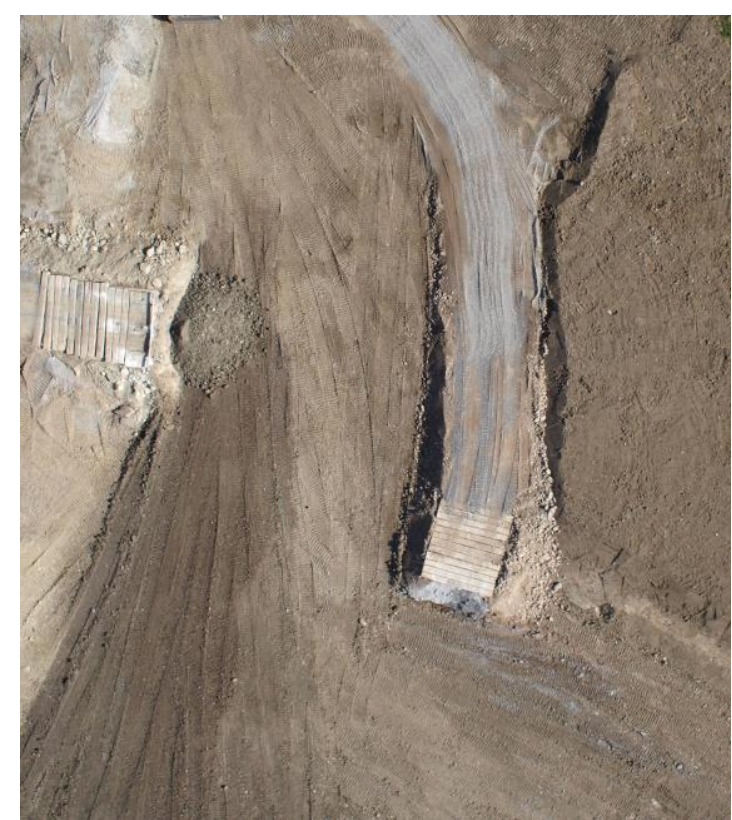

Fig. 2: Subset of an image acquired with the Olympus EP-1 mounted on the microdrones MD4-1000.

\subsection{Camera calibration}

Camera calibration was accomplished using the iWitness software. Using the colour-coded targets (figure 3) for automatic measurements, focal length $\mathrm{c}$, the coordinates of the principal point $\mathrm{x}_{\mathrm{P}}, \mathrm{y}_{\mathrm{P}}$ and the radial lens distortion parameters were estimated within a bundle adjustment. Further parameters of lens distortion were not significant, therefore they were 
neglected. This straightforward procedure takes approximately 1 hour including image acquisition and processing.

Camera calibration was conducted once before the flight, such that possible changes of the parameters cannot be analysed.

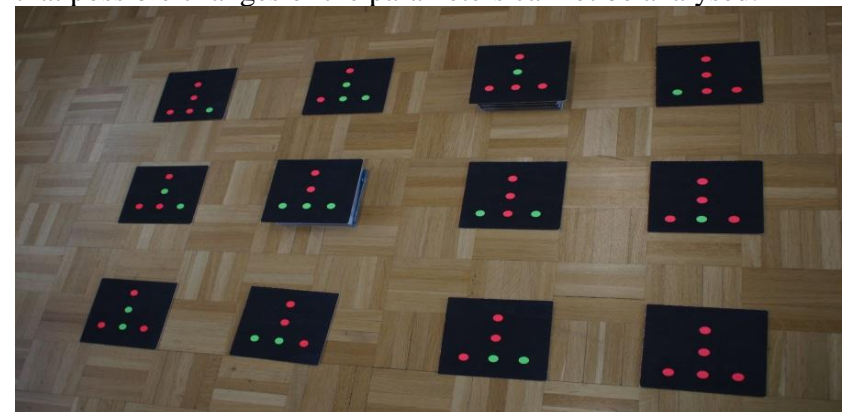

Fig. 3: Red and green colour-coded targets for automatic measurements using the iWitness camera calibration for estimation of the interior orientation parameters.

\subsection{Photogrammetric Processing}

\subsubsection{Image orientation}

Image orientation is one of the most time consuming steps in photogrammetric processing. Using digital cameras, interior orientation parameters can be introduced quite fast as LPS can directly import iWitness calibration results. Exterior orientation requires the measurement of tie points between all neighbouring images, an effort that increases drastically with stereoscopic overlap in and across flight direction and the number of images generally. Setup of the block file in LPS requires ca. 30 minutes.

In LPS, without knowledge of the exterior orientation of the involved images, one would have to measure at least 2 tie points per image pair manually in order to obtain an approximate relative orientation before automatic tie point generation can be started.

The measurement of the GCPs in LPS is supported by the "Calculate image coordinates" function, which after 3 GCPs being measured manually drives the measurement mark closely to the GCP position in the images opened in the viewer. Additionally, it calculates image coordinates in all images the actual GCP is visible. In small areas suited for UAV deployment, a relatively small number of GCPs (6-10) should be sufficient in order to orient the whole block with an acceptable redundancy, therefore this part does not require a significant effort (Table 2).

\subsubsection{DSM generation}

Within the frame of this study, we used the Erdas LPS module eATE (enhanced Automatic Terrain Extraction) for the generation of a high resolution DSM of the gravel pit. The algorithm implemented in eATE works as follows:

- Image matching is performed hierarchically through the image pyramids by means of NCC (Normalized Cross Correlation).

- Matching results were refined by Least Squares Matching (LSM), again through all image pyramids.

- Edge constraint is applied, in this case down to the $3^{\text {rd }}$ image pyramid.

- In the next step, reverse matching with a tolerance threshold was applied.

- The matching candidates were filtered using automatic blunder detection based on principal component analysis (PCA) with a tolerance of the standard deviation of 1 pixel.

- For raster generation the points were interpolated including smoothing and spike removal.

It has to be noted that eATE tries to generate a 3D coordinate for every pixel in the areas of stereoscopic overlap. Simultaneous matching in multiple images can be performed in max. 3 images so far, this function is expected to be enhanced to more images in following software versions.

Although the matching procedure is completely automated, depending on the chosen parameters computing time can vary significantly. The main parameters influencing processing speed are the pyramid level to which LSM and edge constraints are performed, the search and correlation window sizes, reverse matching, the block size in which As eATE makes use of up to 4 processors or cores per software licence, computing time also depends on the deployed hardware. Matching between each image pair or triplet runs as a single thread, each thread can run individually on a different core or processor then.

Subsequently, the matching result has to be verified and if necessary edited. In Erdas LPS, this step can be conducted using the Terrain Editor module, which allows for stereoscopic measurement of points or breaklines, editing of points and other features in order to correct the automatic measurements and to fill gaps (compare Figures 4 and 5, several gaps can be identified, especially in regions with homogeneous and repetitive texture, shadow areas and very steep areas).

The resulting DSM serves then as a basis for volume and volumetric change calculation, which is a process which is not affected by the method of DSM generation in terms of effort and therefore will not be discussed here.

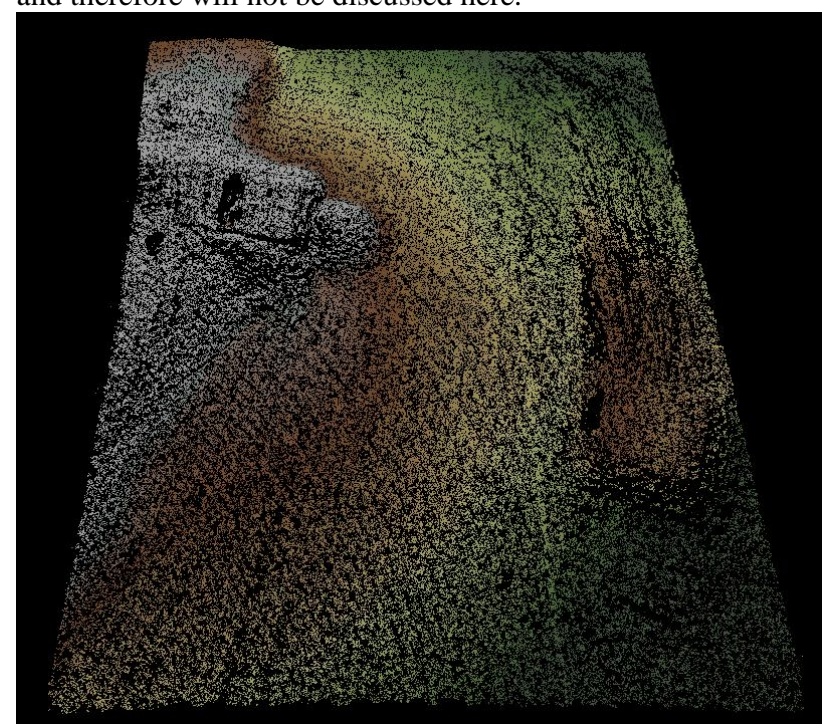

Fig. 4: DSM generated from UAV images. The illustration shows the matched points, colour-coded from green (548 $\mathrm{m}$ above sea level) to grey (556 $\mathrm{m}$ above sea level), the width of the area of interest is ca. $39 \mathrm{~m}$. 


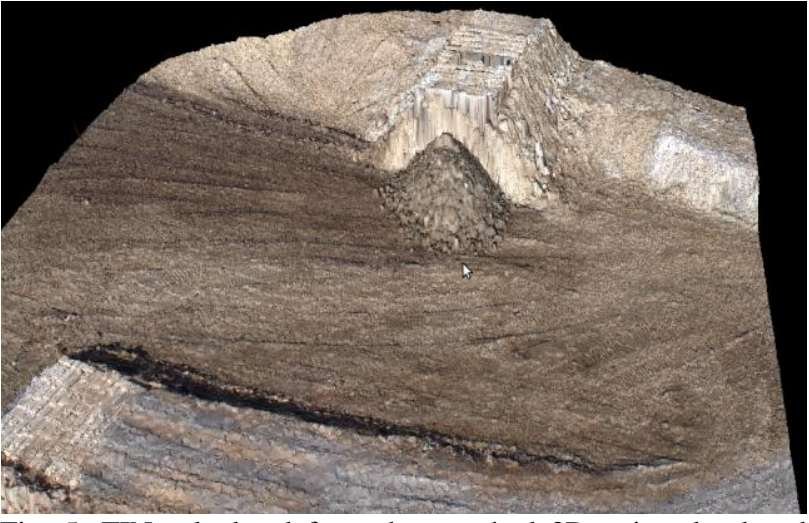

Fig. 5: TIN calculated from the matched 3D point cloud and textured using RGB values from the input images.

\subsubsection{Orthoimage production}

Once an accurate DSM is available, orthoimage production can be performed. This procedure is widely automated and requires human interaction only for input of parameters such as the area of interest and the resolution of the final result. If necessary, the single orthoimages of a photogrammetric block can be mosaicked. Generation of orthomosaics can be more time consuming in case of strong radiometric differences between the input images, which have to be adjusted beforehand.

\subsection{Summary}

Regarding the whole workflow, we acquired the time needed for each step (Table 1) in percent. In table 1, the most time consuming and therefore most costly steps can be identified. Table 1 considers human labour time as well as computing times.

\begin{tabular}{|l|c|}
\hline Step of the workflow & $\begin{array}{l}\text { Time effort in \% of the total } \\
\text { time required }\end{array}$ \\
\hline Flight Planning & 4.1 \\
\hline Data Acquisition & 33 \\
\hline Setup of the Project in LPS & 2 \\
\hline Aerial Triangulation & 11.4 \\
\hline DSM Generation & 41.2 \\
\hline Orthoimage Generation & 1.4 \\
\hline Mosaicking & 6.9 \\
\hline & \\
\hline Total & $\mathbf{1 0 0}$ \\
\hline
\end{tabular}

Table 1: Distribution of time effort for the different steps of UAV photogrammetry (estimation based on a test using 10 images and 10 GCPs).

It can be clearly stated that DSM generation is by far the most time consuming process in our test project, in terms of computing time (ca. 30 minutes per image pair) as well as in terms of editing. The second factor, which is not clearly visible from table 1 , is aerial triangulation (table 2). In our test case, it takes comparably low time for processing due to the small number of images involved, but it has to be taken into account that also in this step, manual quality control and editing are required. Furthermore, before automatic tie point generation can be started, an approximate relative orientation of neighbouring images has to be calculated which in LPS is done based on at least 2 manually measured tie points per image pair (ca. 1 minute per image pair). This effort increases drastically with the number of involved images and the percentages of stereo overlap in and across flight direction. Please note that the percentages of the editing steps (tie points and DSM) will increase subsequently. Additionally, direct georeferencing could help to reduce the amount of mismatches and blunders in tie point generation.

\begin{tabular}{|l|c|}
\hline $\begin{array}{l}\text { Step of Aerial Triangulation } \\
\text { (AT) }\end{array}$ & $\begin{array}{l}\text { Time effort in \% of the time } \\
\text { required for AT }\end{array}$ \\
\hline $\begin{array}{l}\text { Setup of matching parameters } \\
\text { for tie point measurement }\end{array}$ & 3 \\
\hline Measurement of tie points & 1 \\
\hline $\begin{array}{l}\text { Quality control and editing of } \\
\text { tie points }\end{array}$ & 12 \\
\hline GCP measurement in images & 12 \\
\hline Bundle adjustment & 36 \\
\hline Analysis of the results & 36 \\
\hline & $\mathbf{1 0 0}$ \\
\hline Total AT & \\
\hline
\end{tabular}

Table 2: Time effort required for the single steps of aerial triangulation as percentage of the total time needed.

Additionally, a qualitative comparison to competing methods can be conducted. Due to the fact that project requirements can vary in practice in terms of products, topographic characteristics of the area of interest, size of the area of interest, legal conditions (e.g. restrictions concerning navigation in air space such as short distance to an airport), accuracy and resolution of the products to be derived, time required for data acquisition and others more, a generally valid statement cannot be given. Nevertheless, in the following we summarize the main characteristics and possible added values of the different methods.

\subsubsection{GNSS measurements}

GNSS measurement methods feature a rather small overlap of applications with UAV-based photogrammetry. While punctual accuracy of GNSS measurements is comparably high, the point density that can be obtained within a reasonable time is low. However, it is possible to directly produce a DTM instead of a DSM and postprocessing effort is very low. In terms of products that can be derived from both methods, GNSS lacks the option of orthoimages.

\subsubsection{Terrestrial laser scanning}

Terrestrial laser scanning can mainly be used to produce DSMs or DTMs. The point density of the resulting models is in the same order of magnitude and even better than for DSMs resulting from UAV imagery. Additionally, modern laser scanners are often equipped with a digital camera which is calibrated with respect to the laser beam in terms of position and attitude, therefore image information can serve as texture and as a means for additional measurements (monoplotting), e.g. to determine breaklines or object boundaries etc. Data resulting from scans performed on different positions have to be co-registered and geo-referenced, such that additional processing and control point measurements are required. Quality control and manual editing of the measured 3D point clouds usually are required due to erroneous points, the effort is comparable to the effort for editing photogrammetrically derived DSMs. The number of scan positions highly depends on the complexity of the object space to be acquired. Moreover, for the production of high quality orthoimages, additional images from suitable positions above the terrain have to be acquired and processed. Therefore, terrestrial laser scanning in some 
cases could be more efficient than UAV photogrammetry, while in others it might not. Often, it is also the case that a combination of both technologies achieves the optimal result (Eisenbeiss et al., 2005, Remondino et al., 2009).

\subsubsection{Terrestrial photogrammetry}

Terrestrial photogrammetry competes with UAV photogrammetry mainly in the field of documentation of architecture, buildings and other small facilities which feature real 3D geometry. In such cases, the processing steps are more or less identic, the main difference is in data acquisition. UAVs allow the operator to cover also areas that are difficult to access, e.g. roof structures and high buildings. Therefore, both techniques rather complement each other.

2.5.4 Airplane and helicopter based photogrammetry and laser scanning

With increasing size of the area of interest, other airborne platforms such as airplanes and helicopters in combination with medium and large format cameras become strong competitors for UAV platforms. Additionally, fixed-wing UAVs emerged on the market which are capable of covering the same area in a shorter time period, nevertheless they are restricted as well by the line-of-sight limit and therefore always have to operate in the field of visibility of the operator (Leven et al., 2007). Nowadays, small planes equipped with large format cameras can be deployed at relatively low cost, e.g. for gravel pit and waste site documentation. Though the cost for data acquisition is slightly higher than using a mini-UAV if travel cost, operator's salaries and fuel are taken into account, photogrammetric processing becomes much more efficient due to the significantly lower number of images required to cover the same area at the same resolution compared to a small format camera. Finally, according to our experience, using such systems in a project is significantly cheaper than using an UAV equipped with a small format camera. For helicopter- and airplane borne laser scanning, in terms of efficiency the relation to UAV photogrammetry is similar.

\section{CONCLUSIONS}

Our test project has shown that the efficiency of UAV photogrammetry in comparison to other measurement technologies depends on several factors such as the size of the area of interest, which in turn influences the number of involved images. The number of images has a major impact on those steps in the workflow which require manual interaction by a human operator, in particular quality control and editing of tie points and DSM. Although the results obtained for the DSM using LPS eATE are promising, it would be interesting to compare the quality of the automatically generated DSM to results obtained with other software for the same area, even more as LPS eATE was so far not evaluated scientifically concerning its accuracy to our knowledge.

Regardless of the potential to improve efficiency in UAV photogrammetry, it is important to raise awareness of the added value it can provide on the customer side. High resolution orthoimagery and accurate DSMs modelling the topography very detailed on the one hand side, but also the option to produce results with lower resolution and accuracy very rapidly if applicable on the other hand make UAVs the most flexible platform on the market today. Furthermore, UAVs are wellsuited to acquire images in traditional photogrammetric block modes, but also in other configurations

\subsection{Improvement of the workflow}

In order to improve efficiency of the processing workflow, we identified two critical steps that would require further development. On the one hand, being restricted to small format digital compact cameras or SLR cameras, even for projects covering comparably small areas usually a huge amount of images has to be acquired, which in turn increases the effort of image processing. The deployment of medium format cameras could lead to an improvement, although the models available on the market are heavier than small format cameras and would require higher payloads provided by the UAV platform. For example, a Hasselblad H4D-31 medium format camera with a 31 Megapixels sensor weighs $2290 \mathrm{~g}$ while the microdrones MD4-1000 features a maximum payload of $1200 \mathrm{~g}$. Consequently, one would have to use UAVs featuring a larger payload, which in turn are larger and more difficult to operate such that system and operator costs will increase again.

Furthermore, wide angle small format cameras help to decrease the flying altitude, which is important when UAVs have to operate in areas where it is restricted to fly above a certain limit, e.g. $150 \mathrm{~m}$ in areas close to airports.

The second important factor is related to aerial triangulation. If tie point measurement could run fully automated and obtain reliable results, this time consuming step in the processing chain could be accelerated drastically. The problem is influenced by 2 factors: First, a rough relative orientation of the images is needed to enable automatic tie point measurement in LPS. Here, an efficient import tool that assigns the camera's position (X, Y, $Z$ ) and attitude $(\omega, \varphi, \kappa)$ at the time of acquisition to each image could increase efficiency. The second factor is the deployed algorithm for tie point generation, which in case of LPS has to be adapted to the image content using various parameters. Determination of the optimal parameters can also be a time consuming procedure and manual quality control and editing still needs to be conducted after tie point generation. More automated algorithms (Noah et al., 2006, Furukawa and Ponce, 2007, Furukawa and Ponce, 2009) in combination with advanced software for bundle adjustment (e.g. Leica ORIMA) that supports direct georeferencing could increase automation for this procedure as well.

The above mentioned methods estimate the camera parameters on the job by means of self calibration. This can also be an alternative compared to a test field calibration because parameter changes, which can be significant especially for consumer cameras, will not affect the results. Furthermore, it has to be taken into account that a significantly higher number of ground control points would be required for self calibration, which in turn increases the effort of field work. Nevertheless, in a future approach the application of self calibration should be investigated.

\section{REFERENCES}

Demir, N., Eisenbeiss, H. 2010. Report on 3D Modelling of Gravel Pit using UAV images (Phase II). Internal Report of the Institute of Geodesy and Photogrammetry, ETH Zurich, October 2010, 14 pages.

Eisenbeiss, H., 2009. UAV Photogrammetry. PhD Dissertation, ETH Zurich, Nr. 18515, 2009.

Eisenbeiss, H., Lambers, K., Sauerbier, M. and Zhang, L., 2005. Photogrammetric documentation of an archaeological site 
(Palpa, Peru) using an autonomous model helicopter, In: International Archives of the Photogrammetry, Remote Sensing and Spatial Information Sciences, CIPA, Torino, Italy, Vol XXXIV-5/C34, pp. 238-243.

Furukawa, Y., Ponce, J., 2007. Accurate, Dense and Robust Multi-View Stereopsis. In: Computer Vision and Pattern Recognition, 2007, CVPR 2007, IEEE, pp. 1-8.

Furukawa, Y., Ponce, J., 2009. Accurate Camera Calibration from Multi-View Stereo and Bundle Adjustment. International Journal of Computer Vision, 84 (3), pp. 257-268.

Grenzdörffer, G., Engel, A. and Teichert, B., 2008. The Photogrammetric Potential of Low-Cost UAVs in Forestry and Agriculture, In: The International Archives of the Photogrammetry, Remote Sensing and Spatial Information Sciences, ISPRS Congress, Beijing, China, XXXVII. Part B1, pp. 1207-1213.

Lambers, K., Eisenbeiss, H., Sauerbier, M., Kupferschmidt, D., Gaisecker, T., Sotoodeh, S., Hanusch, T., 2007. Combining photogrammetry and laser scanning for the recording and modelling of the late intermediate period site of Pinchango Alto, Palpa, Peru. In: Journal of Archaeological Science, 34, pp. 1702-1710.

Leven, S., Zufferey, J.-C., Floreano, D., 2007. A Simple and Robust Fixed-Wing Platform for Outdoor Flying Robot Experiments. International Symposium on Flying Insects and Robots, Monte Verità, Switzerland, pp. 69-70.

Lötscher, 2011. Lötscher Kies + Beton AG corporate website, http://www.loetscher-kiesbeton.ch/, (accessed July 17 2011).

microdrones, 2011. microdrones corporate website, http://www.microdrones.com/, (accessed July 20 2011).

Niethammer, U., Rothmund, S. and Joswig, M., 2009. UAVbased remote sensing of the slow moving landslide SuperSauze, In: Landslide processes, CERG Editions, Strasbourg, pp. 69-74.

Noah, S., Seitz, S. M., Szeliski, R., 2006. Photo tourism: Exploring image collections in 3D. ACM Transactions on Graphics (Proceedings of SIGGRAPH 2006), 25(3), pp. 835846.

Püschel, H., Sauerbier, M., Eisenbeiss, H., 2008. A 3D model of Castle Landenberg $(\mathrm{CH})$ from combined photogrammetric processing of terrestrial and UAV-based images. In: The International Archives of the Photogrammetry, Remote Sensing and Spatial Information Sciences, Vol. XXXVII, Part B6b, pp. 93-98.

Reidelstuerz, P., Link, J., Graeff, S. and Claupein, W., 2007. UAV (unmanned aerial vehicles) für Präzisionslandwirtschaft. 13. Workshop Computer-Bildanalyse in der Landwirtschaft \& 4. Workshop Precision Farming, In: Bornimer Agrartechnische Berichte, 61, 75-84.

Remondino, F., Gruen, A., von Schwerin, J., Eisenbeiss, H., Rizzi, A., Girardi, S., Sauerbier, M., Richards, H., 2009. MultiSensor 3D Documentation of the Maya Site of Copán. Proceedings of the $22^{\text {nd }}$ CIPA Conference, CIPA, Kyoto, Japan, available online: http://cipa.icomos.org/KYOTO.html.
Vogler, A., Eisenbeiss, H., Aulinger-Leipner, I. and Stamp, P., 2009. Impact of topography on cross-pollination in maize (Zea mays L.). In: European Journal of Agronomy, 31, pp. 99-102.

\section{ACKNOWLEDGEMENT}

The authors thank M. Lötscher (Lötscher Kies \& Beton AG) for his cooperation and support during field work and in discussions as well as D. Novák (ETH Zurich, Chair of Photogrammetry and Remote Sensing) and G. Flury (ETH Zurich) for their efforts during field work. 